\title{
Isolation, Characterization and Myogenic Differentiation of Synovial Mesenchymal Stem Cells
}

\author{
Original \\ Fatma Yassine Meligy ${ }^{1}$, Dalia Abdo Elgamal ${ }^{1}$, Nashwa Ahmed Mohamed Mostafa ${ }^{1}$, \\ Article \\ Ayat sayed Abdelrahman ${ }^{2}$ \\ ${ }^{1}$ Histology and cell biology Department, Faculty of Medicine, Assiut University, Assiut, Egypt \\ ${ }^{2}$ Medical Biochemistry Department, Faculty of Medicine, Assiut University, Assiut, Egypt
}

\begin{abstract}
Background: Synovial membrane represents a worthy source of mesenchymal stem cells (MSCs). Synovial membrane derived stem cells has been described as encouraging line of treatment for musculoskeletal degenratative disorders compared to other sources MSCs. Having higher chondrogenic capacity and being easy to collect without injury of adjacent tissue as well as its proximity to the articular cartilage make it good choice for treatment of such disorders.

Aim of work: The present study aimed to isolate and characterize (MSCs) derived from synovial membrane and to examine its myogenic differentiation potential.

Materials and Methods: four adult mice were used to isolate synovial membrane MSCs using digestion method, after which, 5 -azacytidine (AZA) was used to induce the myogenic differentiation. Stemmness and differentiation characteristics were evaluated by immunocytochemistry (ICC), fluorescence-activated cell sorting (FACS) and real time PCR.

Results: Strong positive expression of CD29, CD44, CD90 and CD105 and negative expression of CD34 and CD45 were reported for MSCs using ICC. Also, FACS analysis showed 92\%,86\%, 93\% and 90\% for CD29,CD44,CD90 and CD105 expressing cells respectively. On the other hand AZA treated cells showed strong desmin and myogen expression ( $80 \%)$.

Conclusion: Synovial mesenchymal MSCs could be expanded in vitro and differentiated towards myogenic linage which is promising treatment strategies in musculoskeletal diseases.
\end{abstract}

Key Words: Mesenchymal stem cells, myogenic differentiation, synovium.

Revised: 08 July 2018, Accepted: 17 December 2018

Corresponding Author: Fatma Y. Meligy, Histology and cell biology department, Faculty of Medicine, Assiut University, Assiut 71516 (Egypt), Tel.: +20 1021900608, Fax: +20882333327, +20882332278 E-mail: fmeligy@gmail.com fmeligy@aun.edu.eg

ISSN: 1110-0559, Vol. 2, No. 1

\section{INTRODUCTION}

Stem cells gained consideration due to their recent use in cell-based therapy in different diseases due to their significant proliferative and differentiation capacities towards various tissue lineages irrespective of their $\operatorname{origin}^{[1]}$. At present, most scientific researches cell based therapy are devoted to development of protocols, preliminary for clinical utilization ${ }^{[2]}$.Adult stem cells, as particularly MSC have been originally isolated from bone marrow $^{[3]}$. Also, they have been retrieved from variety of adult tissues such as muscle ${ }^{[4]}$, dermis ${ }^{[5]}$, adipose tissue ${ }^{[6]}$ and recently synovial membrane $\mathrm{e}^{[7 ; 8 ; 9]}$.

The synovial membrane is a tissue that lines the joint cavity of synovial joints and consists of a lining layer of macrophage-like (type A) and fibroblast-like (type B) synoviocytes and a loose sublining tissue ${ }^{[10]}$.

Proliferation of synoviocytes, fibroblast-like (type B) subsidizes to synovial hyperplasia in response to damage or trauma ${ }^{[11]}$. Accumulated evidence suggested that adult synovial membrane harbors cells that behave like multipotent mesenchymal/stromal stem cells (MSCs) after release and culture expansion ${ }^{[12 ; 13]}$. Rat Synovial
MSCs showed high proliferative potential compared to adipose tissue, bone-marrow, periosteum and musclederived stem cells as shown by higher colony number per each adherent nucleated cell, and cell number per colony ${ }^{[14]}$.

The properties of MSCs can be affected by their preparation as well as by their origin; for instance, stem cells from bone marrow or synovium may better differentiate into chondrogenic and osteogenic lines ${ }^{[15]}$. Hence, the choice of an appropriate stem cell source may also play central role in efficiency of development of a specific tissue regeneration paradigm. For example, Satellite cells showed higher ability to restore functional muscle tissue being of same origin tissue ${ }^{[16]}$.However, because of lack of differentiation of these Satellite cells in higher passages, MSCs were developed as a therapeutic strategy in musculoskeletal diseases ${ }^{[17]}$. MSCs derived from human synovial membranecan restore damaged muscle ${ }^{[12]}$.Although, synovial MSCs were known for their high chondrogenic as well as calcification and adipogenic potentials ${ }^{[15 ; 14]}$ their myogenic differentiation potential have not been well explored. 
So, the aim of our study is to isolate MSCs from synovial membrane and to differentiate these stem cells into skeletal myocytes in vitro.

\section{MATERIALS \& METHODS}

\section{Animals:}

Institutional Animal Care and Use Committee of Assiut University, Faculty of Medicine approved all procedures dealt with mice. Four adult eight-week-old male mice were used in this study as donor animals for establishment of synovial mesenchymal cell culture.

Isolation of synovial MSCs: Primary cells were isolated through excision of the synovial membranes of bilateral knee joints. The tissues were minced, digested for $3 \mathrm{~h}$ at $37 \mathrm{C}^{\circ}$ with type II collagenase $(0.2 \%$; Sigma, Lakewood, N.J., USA) followed by filtration through a $40 \mu \mathrm{M}$ mesh (Becton Dickinson, Franklin Lakes, N.J., USA) to yield single-cell suspensions. Phosphatebuffered saline solution (PBS) was added followed by two rounds of five min centrifugation at $1,500 \mathrm{Xg}$ to eliminate collagenase.

Culture and expansion of synovial MSCs: The liquid fraction containing the cells was centrifuged ${ }^{(18)}$, rinsed twice with Dulbecco's modified Eagle's medium (DMEM) and resuspended in complete culture medium containing Alpha MEM media (Alpha modification of minimal essential medium Eagle) (Biochrom;AG, Berlin, Germany) supplemented with $20 \%(\mathrm{v} / \mathrm{v})$ fetal bovine serum (FBS) (GIBCO BRL) and 1\% (v/v) antibiotic/ antimycotic solution. Isolated cells were plated into flasks and allowed to become adherent.

Detached cells were eliminated by changing the medium every 2 days. The cells were allowed to reach $80 \%$ confluence ( 3 days), after which they were rinsed with serum free DMEM, treated with trypsin-EDTA (GIBCO BRL) for $5 \mathrm{~min}$, washed with DMEM twice, then subcultured in complete DMEM at a density of $2.0 \times$ 105 cells $/ 75 \mathrm{~cm} 2$ dish $^{[19]}$.

\section{Myogenic differentiation:}

Ten mmol /1 DMEM of 5-Azacytidine (AZA) (Sigma Chemical) were added to 50,000 cells of passage 2 synovial MSCs with 10\% FBS and 5\% DHS (Donor horse serum, BiochromAG), and incubated for 24 hours at $37 \mathrm{C}$ and $5 \% \mathrm{CO}^{[20]}$. Untreated cells were considered as controls. The cells were maintained in culture for 28 days. The control stem cells and AZA-treated cells were subsequently analyzed by Fluorescein activated cell sorting (FACS), Immunocytochemistry (ICC) and quantitative polymerase chain reaction (q-PCR) to assess stem cell surface and myogenic markers expression.

\section{Immunocytofluorescence staining:}

The cells of the isolated control and treated group were plated in chamber slides and cultured at $37^{\circ} \mathrm{C}, 5 \% \mathrm{CO} 2$ humidified incubator. After 25- days, the medium was discarded; cells were washed twice with PBS and then fixed in 3\% paraformaldehyde (PFA) in PBS for $20 \mathrm{~min}$ at 37C. PFA was removed and cells were washed three times with $0.1 \%$ Triton $\mathrm{X}-100$ in PBS and incubated for $15 \mathrm{~min}$ at $37 \mathrm{C}$. Cells were incubated for $30 \mathrm{~min}$ at $37 \mathrm{C}$ with $2 \%$ bovine serum albumin (BSA) in PBS to block non specific antigen on the cell surface. Diluted primary antibodies were added to the fixed and blocked cells. The cells were then incubated overnight at $4{ }^{\circ} \mathrm{C}$ with primary antibodies diluted in blocking solution. The following primary antibodies; mouse monoclonal antibodies against CD90, CD 29, CD44 (HCAM), CD45, CD 105 and CD34 (1:200; Santa Cruz Biotechnology, Santa Cruz, CA USA) and rabbit monoclonal antibody against Desmin (1:200; Abcam, Cambridge, UK).

After washing, the cells were mixed with secondary antibody (Alexa Fluor 647)(Invitrogen, CA, USA ) and incubated for another $30 \mathrm{~min}$ on ice. Cells were co-stained with DAPI (1:500; 4', 6-diamidino-2-phenylindole; Molecular Probes, Eugene, OR) to visualize the nuclei. Finally, Stained cells were mounted with fluorescent mounting medium (Dako, Carpinteria, CA).Skeletal muscle from mice served as positive control for all ICC staining. In each group, an isotope control was performed using mouse-IgG1 (Becton Dickinson, San Jose, CA) instead of the primary antibody. The fluorescent images were analyzed and obtained by a fluorescent microscope (Olympus microscope BX51, Japan). ${ }^{[21]}$.

\section{Flow cytometry.}

Cultured isolated control stem cells and AZA treated cells were analyzed using FACS (cell analyzer) (Becton Dickinson Biosciences, Bedford, MA, USA $)^{[22]}$. Cells were suspended in $500 \mu \mathrm{l}$ PBS containing $20 \mathrm{ng} / \mathrm{ml}$ fluorescein isothiocyanate (FITC)-coupled antibodies against CD90, CD45,CD 44, CD 29, CD34, (Santa Cruz Biotechnology), CD105 (Endogin) (Dako Cytomation, Carpinteria, CA) and myogenin (mouse monoclonal, Becton Dickinson Franklin Lakes, N.J., USA), or as an isotype control, FITC-coupled nonspecific IgG (Becton Dickinson). After washing, the cells were incubated for another 30 min away from light on ice with Alexa Fluor 647 (Santa Cruz Biotechnology) as a secondary antibody. Then, the cells were washed twice and resuspended in 1 $\mathrm{ml}$ PBS for analysis.

\section{RNA extraction and real-time PCR analysis:}

RNA extraction and real-time PCR analysis: Total RNA was extracted from cells and purified using Trizol reagent (Invitrogen, Carlsbad, CA) in accordance with the manufacturer's protocol (Invitrogen, Carlsbad, CA). High Capacity cDNA reverse transcription kit (Applied Biosystems, Foster City, CA,USA) was used to synthesize the $1^{\text {st }}$ strand cDNA from500ng of total RNA and random hexamer. Real-time RT-PCR was performed in a StepOne ${ }^{\mathrm{TM}}$ realtime PCR System (Applied BioSystems) using syber green for desmin and $\mathrm{b}$ actin as an internal 
control. The following primers were used, desmin forward primer 5'-.TACACCTGCGAGATTGATGC-3'. desmin reverse primer: 5'-GTAGCCTCGCTGACAACCTC-3', and B-actin primer as ainternal control, forward 5'-GGACTTCGAGCAAGAGATGG-3 and reverse 5'-AGCACTGTGTTGGCGTACAG-3.

Statistical analysis of the data: Statistical significant differences between the two groups were determined using Student's t test. All data are presented as mean \pm SD is an index of the variability of the original data points. Statistical significance was set at $\mathrm{P}<0.05$.

\section{RESULTS:}

\section{Microscopic cell morphology:}

Synovial membrane derived Stem cells were plasticadherent and demonstrated characteristic spindle-shaped and fibroblast-like morphology. The cells reach $80 \%$ confluence in one week (Fig. 1).

After induction of differentiation, the cells were observed every 2 days for 28 days, the cells were analyzed with fluorescence and inverted microscopy to evaluate the growth and myogenic differentiation of both groups.

\section{Phenotypic characterization \&Immunocytochemistry}

Synovial membrane derived MSCs. showed high expression of CD29, CD44, CD90, and CD105 surface markers (Fig.2A,B,C,D), while the immunophenotypes were noted negative for CD34 and CD45; markers of hematopoietic progenitors and endothelial cells respectively(Fig. $2 \mathrm{E}$ and $\mathrm{F}$ ). As expected synovial membrane derived MSCs expressed weak desmin (Fig. 3a). Interestingly, the AZA-treated cells showed strong expression of desmin protein (Fig 3b).

\section{Fluorescence-activated cell sorting analysis}

Flowcytometric analysis of synovial membrane derived mesenchymal cells demonstrated positive expression for CD29, CD44, CD 90 and CD105 (Fig 4a: $\mathrm{B}, \mathrm{C}, \mathrm{D}, \mathrm{E})$ and negative expression for CD34 and $\mathrm{CD}$ 45 (Fig.4a: F and G). The percentage of CD29, CD44, CD 90 and CD105- positive-expressing cells was $92 \%$, $86 \%, 93 \%$ and $90 \%$ respectively. In addition, myogenin expression was statictically higher in AZA-treated MSCs (T) $(80 \%)$ compared to the untreated synovial derived -MSCs(C) (12\%) (Fig.4b: b).

\section{Reverse transcription polymerase chain reaction}

Desmin expression was detected weekly in myogenic differentiated cells throughout observation period by PCR analysis (unpublished results). However, Desmin expression in AZA-treated cells was higher than that

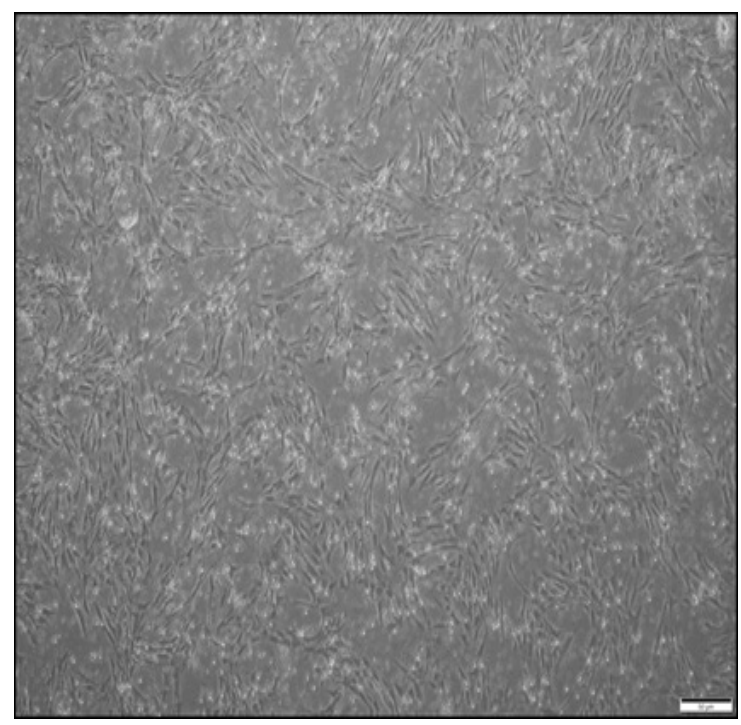

Fig.1 Growth morphology of isolated MSCs derived from the synovial membrane. They are spindle in shape and fibroblast like. 

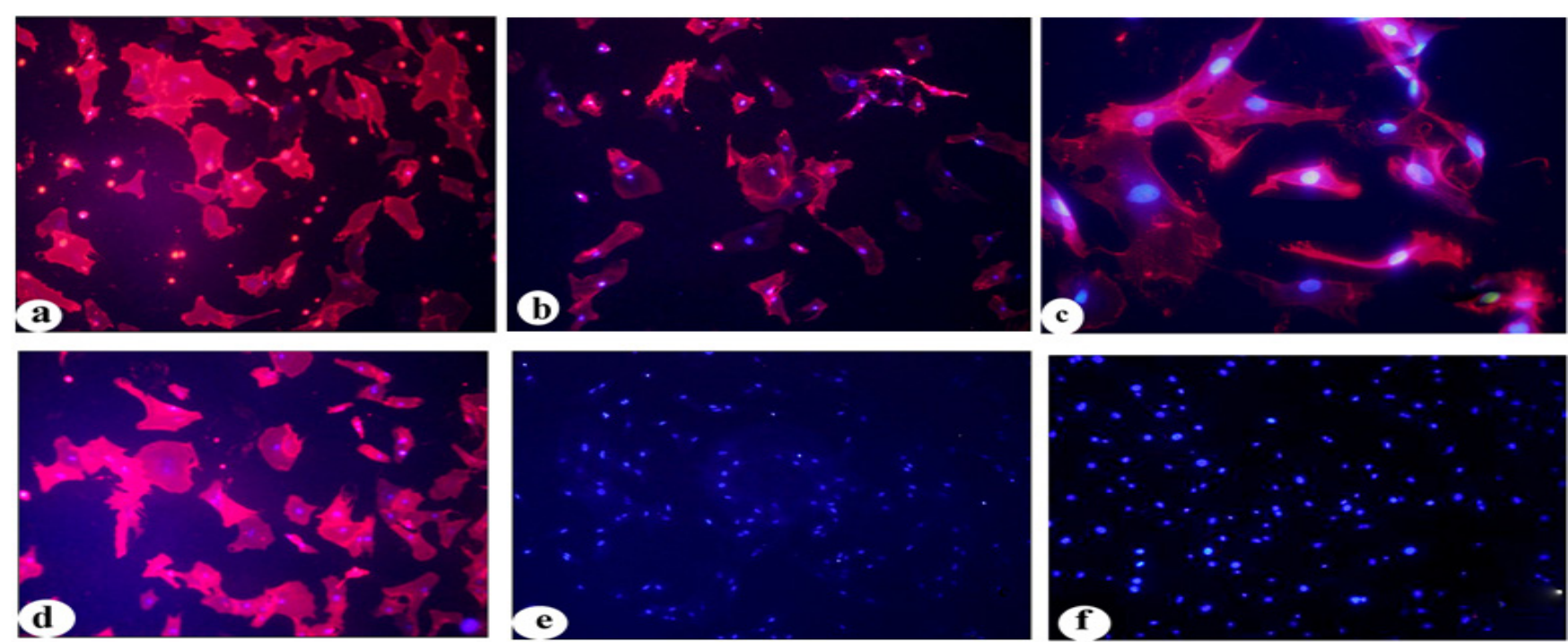

Fig.2 Immunofluorescence staining for merged DAPI stained nuclei (blue); CD 29, CD 44,; CD 90, CD 105;CD 34,CD45, secondary antibody is Alexa Flour 647 (red), in isolated MSCs derived from synovial membrane were evaluated by fluorescent microscope. In (A-D) MSCs relevant to CD29, CD 44, CD 90 and CD 105 positive expression are detected respectively. However, In (E-F) MSCs relevant to CD 34, CD 45 negative expressions are detected, respectively.
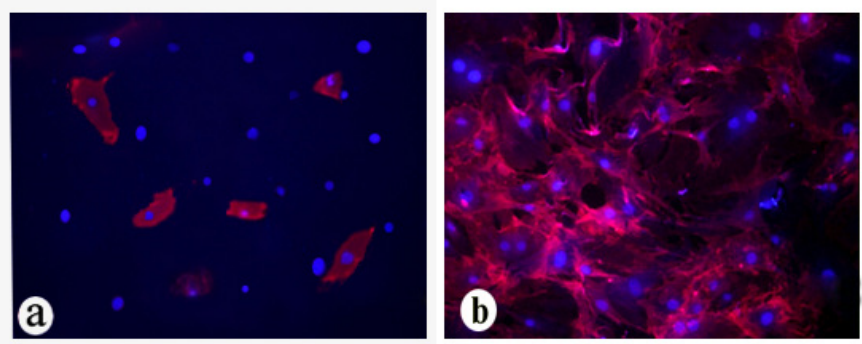

Fig.3 Immunofluorescence staining for merged DAPI-stained nuclei (blue) and desmin-Alexa Flour 647(red) were evaluated by inverted microscopy. In (a), weak expression of desmin in the cytoplasm of few control untreated MSCs, While in (b), significant positive expression of desmin in the cytoplasm of treated MSCs derived from synovial membrane, were detected 4wk after induction of differentiation.
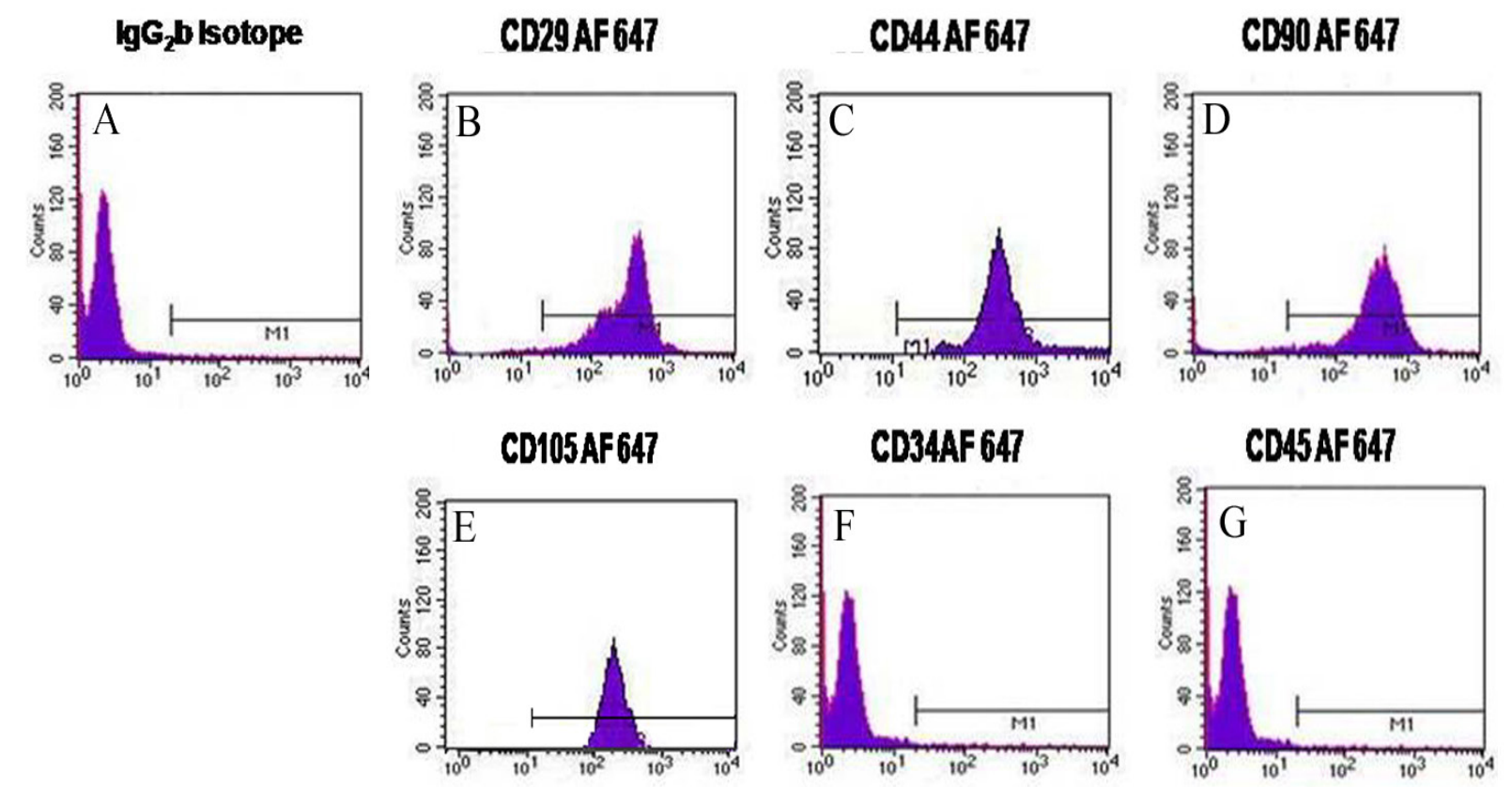

Fig.4a Expression of MSCs surface markers are measured by flowcytometry in MSCs derived from synovial membrane. (a) Representative plot of IgG matched isotope (plot; A), CD29 (plot; B), CD44 (plot; C), CD90 (plot; D), CD105 (plot; E) CD34 (plot; f) and CD45 (plot G). 

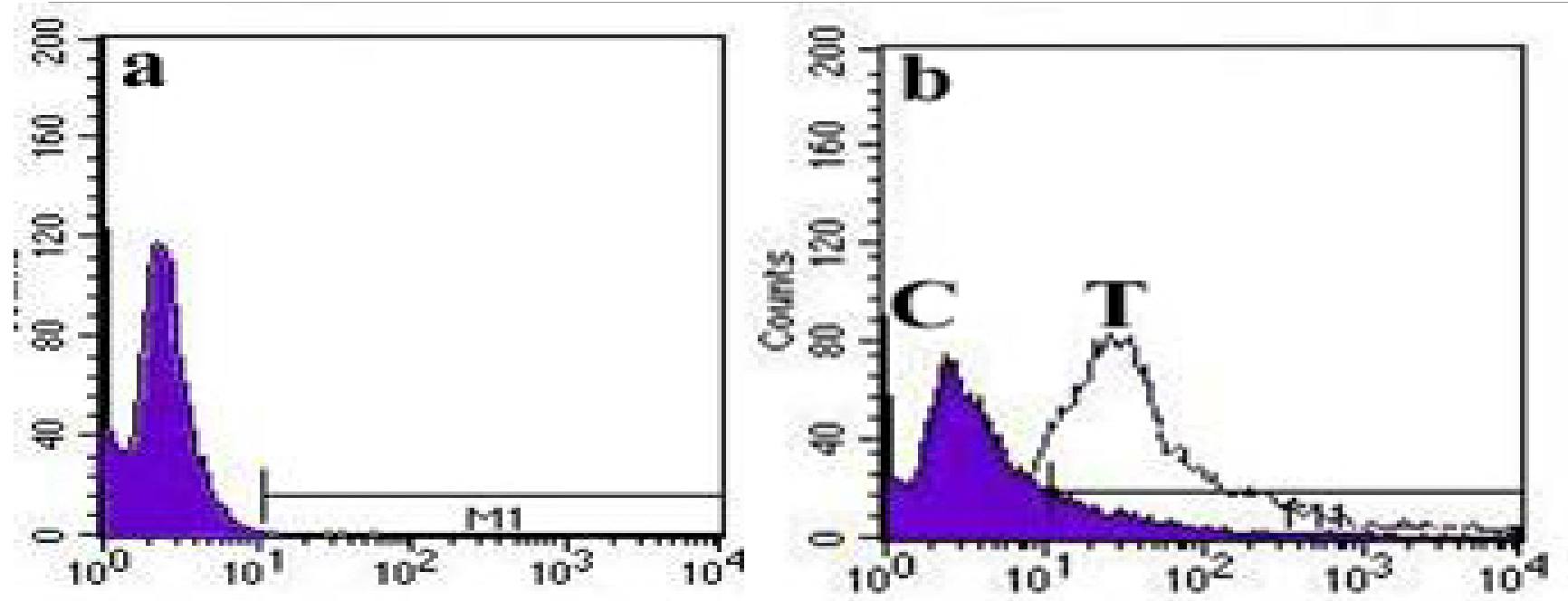

Fig.4b Expression of striated muscle marker myogenin was measured by flowcytometry in SM-MSCs and in 5-azacytidine-treated SM-MSCs after induction of myogenic differentiation. (a) Representative plot of IgG2b Isotope.(b) Representative of myogenin expression after 7 days in control (C) and in 5-azacytidine treated SD- MSCs(T)

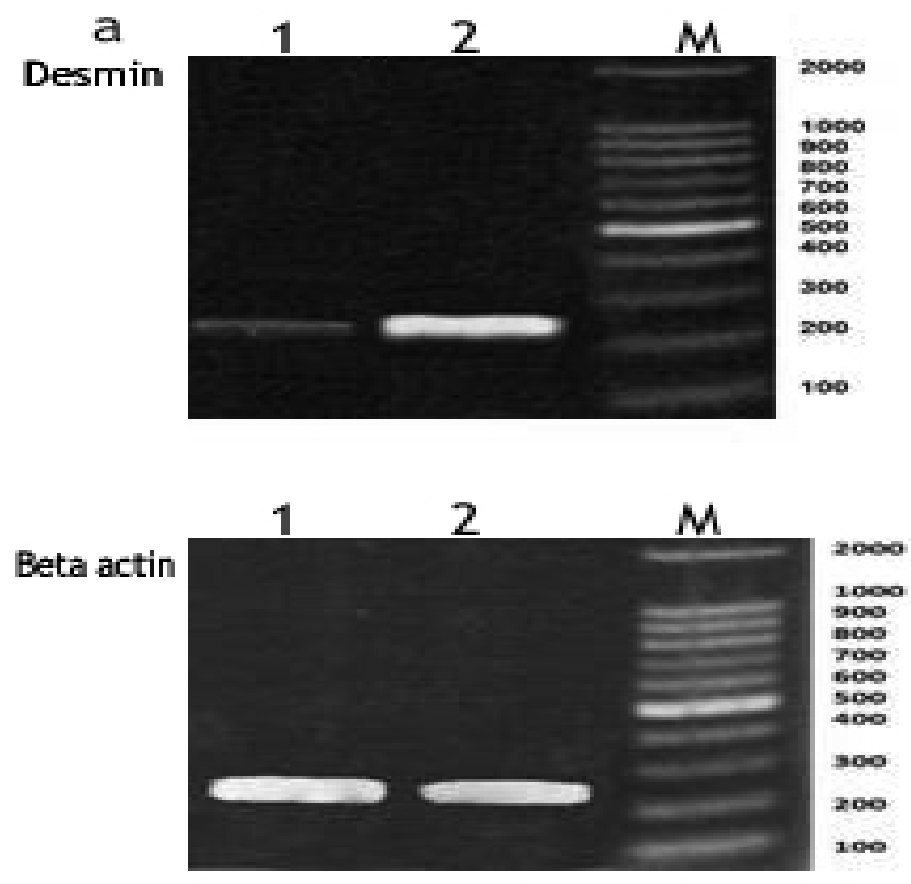

Fig5a. Expression of striated muscle marker desmin in untreated and 5-azacytidine-treated MSCs derived from synovial membrane 4 weeks after induction of myogenic differentiation. Representative PCR analysis of desmin and $\beta$-actin mRNA expression in untreated (lane 1) and 5-azacytidine-treated (lane 2)MSCs derived from synovial membrane. 


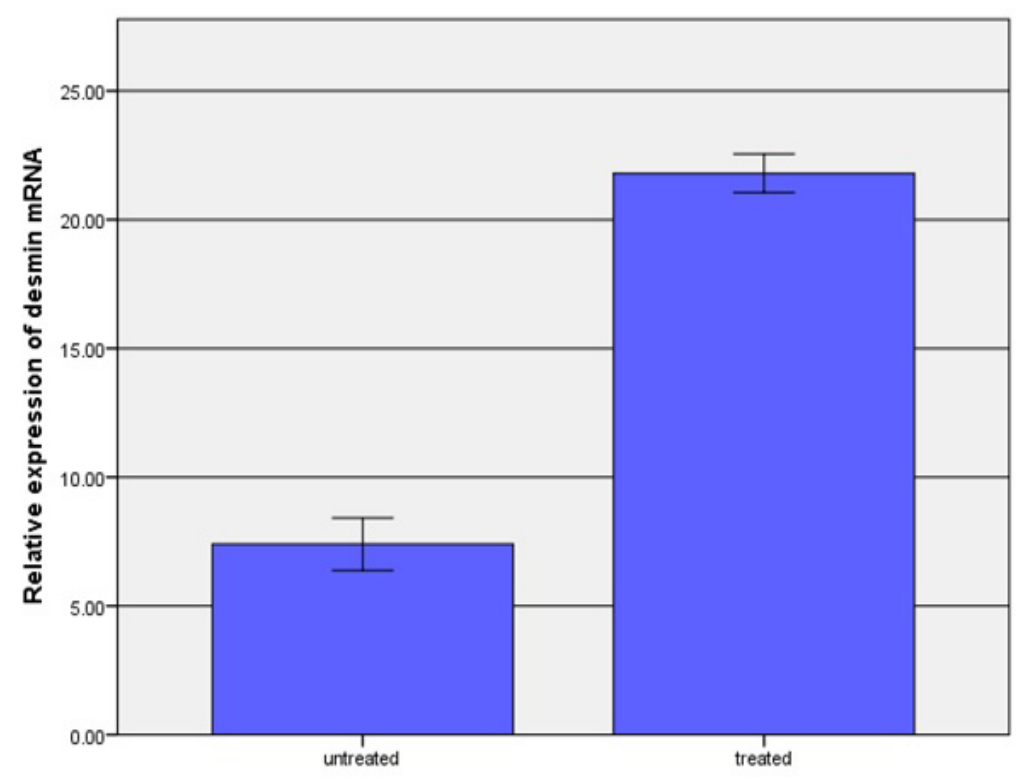

Fig5b Summarized data of relative mRNA level of desmin in untreated and 5-azacytidine-treated MSCs derived from synovial membrane 4weeks after induction of myogenic differentiation. $\beta$-actin was used as the endogenous RNA control to normalize for differences in the amount of total RNA.

in untreated control cells (Fig. 5a).RT-PCR analysis demonstrated further characterization of myogenesis, where AZA-treated SM-MSCs had significantly higher Desmin mRNA expression than control SM-MSCs with its peak at the 28th day (Fig.5b).

\section{DISCUSSION}

Neuromuscular disease encompasses both intrinsic muscle pathology and nerve pathology distorting the function of the muscles. Currently, gene and cell therapy are recognized as putative treatment strategies for such neuromuscular diseases ${ }^{[23]}$. In this study, we examined the potential of mice synovial membrane derived mesenchymal stem cells ( SM-MSCs) to undergo myogenic differentiation in vitro. Morphology, mRNA and protein markers were investigated to show the true myogenic differentiation.

It is particularly noteworthy that the synovium has a high regenerative capacity, and it is easy to be obtained arthroscopically with minimal invasiveness. Besides, a least amount of synovium is sufficient to extract the MSCs successfully ${ }^{[24]}$. Also, Synovial membrane-derived cells were known to have the highest chondrogenic potential that last after many passages ${ }^{[14]}$, employing pluripotent character of the synoviocytes. These pluripotent synoviocyte scan be isolated and expanded ex vivo, thereby providing a unique and accessible population of pluripotent cells from an unexpected tissue resource $^{(7)}$. Little is known about immunophenotypic, structural properties of these cells. Therefore, phenotypic and myogenic differentiation features of these cells were investigated in this study.

In this study, we were able to isolate SM-MSCs from synovial membrane of mice using the enzyme digestion method. Cells were cultured and purified by their adherent ability, colony formation and uniform morphology. In vitro cultivated SM-MSCs expressed cell-surface markers according to the criteria reported by Mesenchymal and Tissue Stem Cell Committee of the International Society for Cellular Therapy including CD29, CD44, CD90 and CD105 $(25,26,27)$. SM-MSCs cells were also lacking the expression of CD34 and CD45, a cell-surface marker associated with lymphohematopoietic cells as shown by both FACS and ICC. These results indicated the high selfrenewal capacity of the synovium-derived MSCs in both $\operatorname{rat}^{[28,29]}$ and human ${ }^{[12]}$. Also it have noted that these cells still hold the same kinetics of growth up to passages $10^{[12]}$.

To further show the myogenic potential different ion of the SM-MSCs AZA were added for $24 \mathrm{~h}$ and the expression of myoblast markers myogenin and desmin were analyzed weekly untill day 28 from induction of differentiation. 
According to the FACS analysis, the expression peak of skeletal muscle marker myogenin was at the end of first week where it reached $80 \%$ in differentiated SM-MSCs. After which myogenin expression decreased. This pattern of myogenin expression in SM-MSCs was similar to

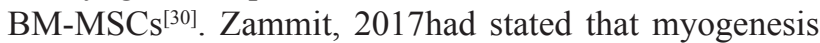
is regulated by a group of transcription factors as MyoD and Myf5 and myogenin, where MyoD and Myf5 are involved in the establishment of the skeletal muscle lineage ${ }^{[31]}$ while myogenin is required for fusion and terminal differentiation ${ }^{[32,33]}$. So, myogenin is expressed in late period of myogenic differentiation, and this explain the peak of myogenin at $7 \mathrm{~d}$ followed by plateau levels of mature muscle markers.

In the meantime, expression of desmin in AZA-treated cells was higher than that in untreated control cells but the peak was at the $28^{\text {th }} \mathrm{d}$. Expression of muscle-specific desmin with an up-regulation of this key initial transcription factor is characteristic in myoblasts and myocytes as musclespecific marker gene and it is common to be used to detect cells with myogenic potential ${ }^{[33]}$. Expression of specific skeletal muscle markers such as desmin, alfa-actin, sarcomeric actin and myogenin appeared following transdifferentiation process. Moreover, a previous study showed that early implanted human SM-MSCs in a model of muscle injury persisted within the regenerated muscle as quiescent satellite cells. Therefore, it has been proved that human SM-MSCs can participate in skeletal muscle regeneration in vivo by long term persistence and contribution to both myofibers and functional satellite cells ${ }^{[13]}$.

Mogenic differentiation of human SM-MSCs have been characterized in an in vivo model of skeletal muscle regeneration and provided evidence of their capacity to partially restore specific pathophysiologic features of the dystrophic muscle in the mdx mouse model of muscle dystrophy ${ }^{[13]}$.Although similar results were observed when MSCs were harvested from bone marrow and cocultured with muscle precursor cells, previous authors had questioned the role of AZA as there are some evidences that it slightly increases myogenic differentiation. They claimed that the strongest effect on MSCs differentiation had occurred by adding conditioned medium from muscle precursor cells ${ }^{[34]}$.

The present data further supports previous findings that the Synovial mesenchymal stem cells could be expanded in vitro and induced to myogenic differentiation which appears to be a promising in treatment strategies in musculoskeletal diseases.

\section{CONFLICT OF INTEREST}

The authors have no conflict of interest.

\section{References}

1. Takahashi K, Yamanaka S. "Induction of pluripotent stem cells from mouse embryonic and adult fibroblast cultures by defined factors".Cell. 2006 Aug 25;126(4):663 - 76
2. McGonagle, Dennis, Cosimo De Bari, Peter Arnold, and Elena Jones. "Lessons from Musculoskeletal Stem Cell Research: The Key to Successful Regenerative Medicine Development." Arthritis \& Rheumatology 56, no. 3 (2007): 714 - 21.

3. Alessio Giai Via, Antonio Frizziero, and Francesco Oliva. "Biological properties of mesenchymal Stem Cells from different sources. " Muscles Ligaments Tendons J. 2012 Jul-Sep; 2(3): 154-162

4. Meregalli M, Farini A and Torrente Y"Mesenchymal Stem Cells as Muscle Reservoir"J Stem Cell Res Ther 1:105. Volume 1 • Issue 2 (2011)

5. Gao, Yuhua, Chunyu Bai, Hui Xiong, Qian Li, Zhiqiang Shan, Linsheng Huang, Yuehui Ma, and Weijun Guan. "Isolation and Characterization of Chicken Dermis-Derived Mesenchymal Stem/ Progenitor Cells." BioMed research international (2013), 626258.

6. Wu, Ling, Xiaoxiao Cai, Shu Zhang, Marcel Karperien, and Yunfeng Lin. "Regeneration of Articular Cartilage by Adipose Tissue Derived Mesenchymal Stem Cells: Perspectives from Stem Cell Biology and Molecular Medicine." Journal of cellular physiology 228, no. 5 (2013): 938 - 44.

7. Koyama, Noriaki, Yasunori Okubo, Kazumasa Nakao, Kenji Osawa, Kazuma Fujimura, and Kazuhisa Bessho. "Pluripotency of Mesenchymal Cells Derived from Synovial Fluid in Patients with Temporomandibular Joint Disorder." Life sciences 89 , no. 1947-741: 2011 20-.

8. Liu, Zhiming, Xing Long, Jian Li, Lili Wei, Zhongcheng Gong, and Wei Fang. "Differentiation of Temporomandibular Joint Synovial Mesenchymal Stem Cells into Neuronal Cells in Vitro: An in Vitro Study." Cell biology international 35, no. 1 (2011): 87 - 91.

9. Jones, Brendan A, and Ming Pei. "Synovium-Derived Stem Cells: A Tissue-Specific Stem Cell for Cartilage Engineering and Regeneration." Tissue Engineering Part B: Reviews 18, no. 4 (2012): 301 - 11.

10. Bartok, Beatrix, and Gary S Firestein. "FibroblastLike Synoviocytes: Key Effector Cells in Rheumatoid Arthritis." Immunological reviews 233, no. 1 (2010): 233 - 55.

11. Caroline Ospelt "Synovial fibroblasts in 2017"RMD Open. 2017; 3(2): e000471

12. Anke J. Roelofs, Janja Zupan1, Anna H.K. Riemen , Karolina Kania , Sharon Ansboro , Nathan White, Susan M. Clark \& De Bari ,Cosimo "Joint morphogenetic cells in the adult mammalian synovium" .Nature Communications volume8, Article number: 15040 (2017). 
13. De Bari, Cosimo, Francesco Dell'Accio, and Frank $\mathrm{P}$ Luyten. "Failure of in VitroDifferentiated Mesenchymal Stem Cells from the Synovial Membrane to Form Ectopic Stable Cartilage in Vivo." Arthritis \& Rheumatology 50, no. 1 (2004): 142 - 50.

14. Yoshimura, Hideya, Takeshi Muneta, Akimoto Nimura, Akiko Yokoyama, Hideyuki Koga, and Ichiro Sekiya. "Comparison of Rat Mesenchymal Stem Cells Derived from Bone Marrow, Synovium, Periosteum, Adipose Tissue, and Muscle." Cell and tissue research 327, no. 3 (2007): 449 - 62.

15. Sakaguchi, Yusuke, Ichiro Sekiya, Kazuyoshi Yagishita, and Takeshi Muneta. "Comparison of Human Stem Cells Derived from Various Mesenchymal Tissues: Superiority of Synovium as a Cell Source." Arthritis \& Rheumatology 52, no. 8 (2005): 2521 - 29.

16. Boonen, Kristel JM, and Mark J Post. "The Muscle Stem Cell Niche: Regulation of Satellite Cells During Regeneration." Tissue Engineering Part B: Reviews 14, no. 4 (2008): 419 - 31.

17. Carlson, Morgan E, and Irina M Conboy. "Loss of Stem Cell Regenerative Capacity within Aged Niches." Aging cell 6, no. 3 (2007): 371 - 82.

18. Karystinou, Alexandra, Francesco Dell'Accio, Tobias BA Kurth, Henning Wackerhage, Ilyas M Khan, Charles W Archer, Elena A Jones, Thimios A Mitsiadis, and Cosimo De Bari. "Distinct Mesenchymal Progenitor Cell Subsets in the Adult Human Synovium." Rheumatology 48, no. 9 (2009): 1057 - 64.

19. Harvanová, D, T Tóthová, M Sarisský, J Amrichová, and J Rosocha. "Isolation and Characterization of Synovial Mesenchymal Stem Cells." Folia biologica 57, no. 3 (2011): 119.

20. Drost, Adriana C, Sibylle Weng, Gerhard Feil, Jochen Schäfer, Simon Baumann, Lothar Kanz, Karl-Dietrich Sievert, Arnulf Stenzl, and Robert Möhle. "In Vitro Myogenic Differentiation of Human Bone Marrow-Derived Mesenchymal Stem Cells as a Potential Treatment for Urethral Sphincter Muscle Repair." Annals of the New York Academy of Sciences 1176, no. 1 (2009): $135-43$.

21. Fatma Y. Meligy \& Katsumi Shigemura \& Hosny M. Behnsawy \& Masato Fujisawa \&Masato Kawabata \& Toshiro Shirakawa.The efficiency of in vitro isolation and myogenic differentiation of MSCs derived from adipose connective tissue, bone marrow, and skeletal muscle tissue.. In Vitro Cell.Dev.Biol.-Animal 48:203-215 DOI 10.1007/s116269488--012-x (2012)
22. Yamamoto, Naoki, Hirohiko Akamatsu, Seiji Hasegawa, Takaaki Yamada, Satoru Nakata, Mahito Ohkuma, Ei-Ichi Miyachi, Tohru Marunouchi, and Kayoko Matsunaga. "Isolation of Multipotent Stem Cells from Mouse Adipose Tissue." Journal of dermatological science 48, no. 1 (2007): 43 - 52.

23. Meregalli, Mirella, Andrea Farini, and Yvan Torrente. "Stem Cell Therapy for Neuromuscular Diseases." In Stem Cells in Clinic and Research: InTech, 2011.

24. Qi, Jun, Anmin Chen, Hongbo You, Kunpeng Li, Di Zhang, and Fengjing Guo. "Proliferation and Chondrogenic Differentiation of Cd105-Positive Enriched Rat Synovium-Derived Mesenchymal Stem Cells in Three-Dimensional Porous Scaffolds." Biomedical Materials 6, no. 1 (2011): 015006.

25. Donzelli, E, A Salvade, P Mimo, M Vigano, M Morrone, R Papagna, F Carini, et al. "Mesenchymal Stem Cells Cultured on a Collagen Scaffold: In Vitro Osteogenic Differentiation." Archives of oral biology 52, no. 1 (2007): 64 - 73.

26. Yu, Yue, Ai-Hua Yao, Nian Chen, Li-Yong Pu, Ye Fan, Ling Lv, Bei-Cheng Sun, Guo-Qiang Li, and Xue-Hao Wang. "Mesenchymal Stem Cells overExpressing Hepatocyte Growth Factor Improve Small-for-Size Liver Grafts Regeneration." Molecular Therapy 15, no. 7 (2007): 1382 - 89.

27. de Macedo Braga, Luisa Maria Gomes, Silvia Lacchini, Beatriz D'Agord Schaan, Bruno Rodrigues, Kaleizu Rosa, Kátia De Angelis, Luciano Figueiredo Borges, Maria Cláudia Irigoyen, and Nance Beyer Nardi. "In Situ Delivery of Bone Marrow Cells and Mesenchymal Stem Cells Improves Cardiovascular Function in Hypertensive Rats Submitted to Myocardial Infarction." Journal of biomedical science 15, no. 3 (2008): 365 - 74.

28. Jian-Feng Pan, Shuo Li, Chang-An Guo , DuLiang Xu, Feng Zhang, Zuo-Qin Yan and Xiu-Mei Mo."Evaluation ofsynovium-derivedmesenchymal stem cells and 3D printed nanocomposite scaffolds for tissue engineering."Sci. Technol. Adv. Mater. 2015 Aug; 16(4): 045001

29. Dezawa, Mari, Hiroto Ishikawa, Yutaka Itokazu, Tomoyuki Yoshihara, Mikio Hoshino, Shin-ichi Takeda, Chizuka Ide, and Yo-ichi Nabeshima. "Bone Marrow Stromal Cells Generate Muscle Cells and Repair Muscle Degeneration." Science 309, no. 5732 (2005): 314 - 17.

30. Gang, Eun Ji, Ju Ah Jeong, Seung Hyun Hong, Soo Han Hwang, Seong Whan Kim, Il Ho Yang, Chiyoung Ahn, Hoon Han, and Hoeon Kim. "Skeletal Myogenic Differentiation of Mesenchymal Stem Cells Isolated from Human Umbilical Cord Blood." Stem cells 22, no. 4 (2004): 617 - 24. 
31. Zammit PS. "Function of the myogenic regulatory factors Myf5, MyoD, Myogenin and MRF4 in skeletal muscle, satellite cells and regenerative myogenesis". Semin Cell Dev Biol. 2017 Dec;72:19 - 32.

32. Nikolaos P. Mastroyiannopoulos , Paschalis Nicolaou , Mustafa Anayasa, James B. Uney, Leonidas A. Phylactou . "Down-Regulation of Myogenin Can Reverse Terminal Muscle Cell Differentiation . "PLoS One. 2012; 7(1): e29896.

33. Beier, Justus P, Franz F Bitto, Claudia Lange, Dorothee Klumpp, Andreas Arkudas, Oliver Bleiziffer, Anja M Boos, Raymund E Horch, and Ulrich Kneser. "Myogenic Differentiation of Mesenchymal Stem Cells Co-Cultured with Primary Myoblasts." Cell biology international 35, no. 4 (2011): 397 - 406.
34. Bajek, Anna, Tomasz Drewa, Romana Joachimiak, Zaneta Spoz, Maciej Gagat, Magdalena Bodnar, Robert Debski, Alina Grzanka, and Andrzej Marszalek. "Myogenic Differentiation of Mesenchymal Stem Cells Is Induced by Striated Muscle Influences in Vitro." Current Signal Transduction Therapy 7, no. 3 (2012): 220 - 27. 
العزل والتوصيف والتمايز العضلي للخلايا الجذعية الميزنشيمية المستخلصه من الغثاءاءلزليلي الئي

فاطمة ياسبن مليجي - داليا عبده الجمل ـ نشوى أحمد محمد مصطقى ـ آيات سيد عبد الرحمن 1قسم الهستولوجى 2قسم الكيمياء الحيويه- كلية الطب -جامعة اسيوط -مصر ملخص البحث

الخلفية: يمثل الغثاء الزليلي مصدر ا قيما للخلايا الجذعية الميزنشيمية .وقد وصفت الخلايا الجذعية المستمدة من الغثاء الزليلي بانها طريقة واعدة لعلاج اضطر ابات

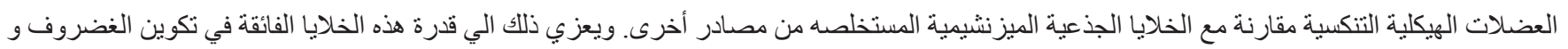

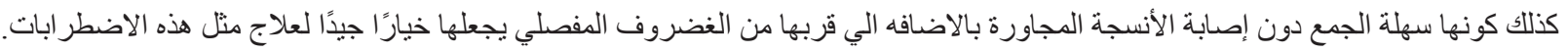

الهرف من البحث: هدفت الدر اسة إلى عزل وتوصيف الخلايا الجذعية الميزنشيمية من الغشاء الزليلي وفحص إمكانات التمايز العضلي.المو اد و الطرق: تم استخدام

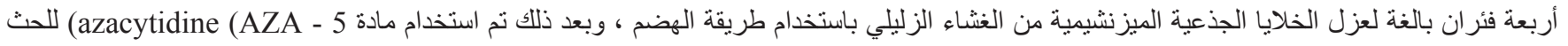

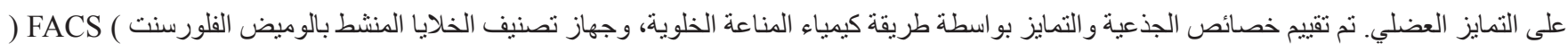
وتفاعل PCR محدد الزمن.

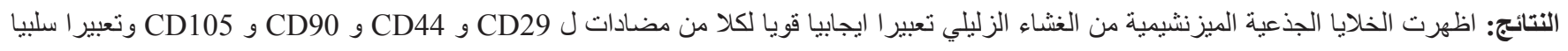

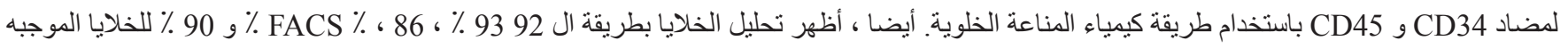

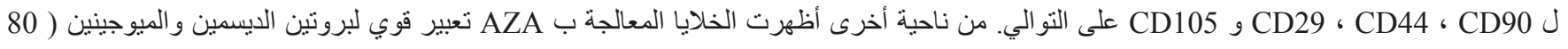

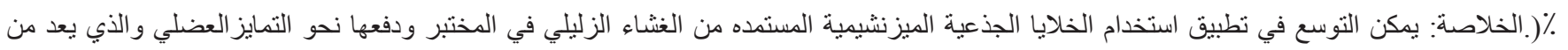

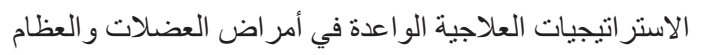

